\title{
OPEN Abnormal composition of microbiota in the gut and skin of imiquimod-treated mice
}

\begin{abstract}
Hiroyo Shinno-Hashimoto ${ }^{1,2}$, Yaeko Hashimoto ${ }^{2,3}$, Yan Wei ${ }^{2,4}$, Lijia Chang ${ }^{2}$, Yuko Fujita ${ }^{2}$, Tamaki Ishima², Hiroyuki Matsue ${ }^{1} \&$ Kenji Hashimoto ${ }^{2 \bowtie}$

Psoriasis is a chronic, inflammatory skin disease. Although the precise etiology of psoriasis remains unclear, gut-microbiota axis might play a role in the pathogenesis of the disease. Here we investigated whether the composition of microbiota in the intestine and skin is altered in the imiquimod (IMO)treated mouse model of psoriasis. Topical application of IMQ to back skin caused significant changes in the composition of microbiota in the intestine and skin of IMQ-treated mice compared to control mice. The LEfSe algorithm identified the species Staphylococcus lentus as potential skin microbial marker for IMO group. Furthermore, there were correlations for several microbes between the intestine and skin, suggesting a role of skin-gut-microbiota in IMQ-treated mice. Levels of succinic acid and lactic acid in feces from IMQ-treated mice were significantly higher than control mice. Moreover, the predictive functional analysis of the microbiota in the intestine and skin showed that IMQ caused alterations in several KEGG pathways. In conclusion, the current data indicated that topical application with IMQ to skin alters the composition of the microbiota in the gut and skin of host. It is likely that skin-gut microbiota axis plays a role in pathogenesis of psoriasis.
\end{abstract}

Psoriasis is a common chronic inflammatory disease of skin. A recent systematic review reports that the prevalence of psoriasis in children and adults ranged from 0 to $1.37 \%$ and 0.51 to $11.43 \%$, respectively ${ }^{1}$. Furthermore, psoriasis is associated strongly with depression, cardiovascular disease, diabetes, and metabolic syndrome, which influence quality of life in patients ${ }^{2-6}$. Although excessive activation of the immune system plays a major role in the pathogenesis of psoriasis, the precise pathogenesis of this disease remains unclear ${ }^{3,6}$.

Accumulating evidence suggests the physiological and metabolic roles of the microbiota in human health and diseases ${ }^{7-13}$. It is well known that microbes are colonized in the gastrointestinal tract, skin, oral mucosa, vagina and airway in the human, and that the colon is the most abundant site for microbes, followed by the skin $^{14}$. Altered composition of gut microbiota in patients with psoriasis has been reported ${ }^{15-19}$. Furthermore, there are also several reports showing abnormal composition of skin microbiota in patients with psoriasis ${ }^{20-25}$. These findings suggest that alterations in the microbiome in the intestine and on the skin might play a role in the pathogenesis of psoriasis, and that improvement of altered composition of microbiota could be a therapeutic approach for this disease ${ }^{14}$.

Imiquimod (IMQ), a Toll-like receptor 7 agonist, has been used widely as mouse model of psoriasis to understand the inflammatory responses of the $\operatorname{skin}^{26,27}$. It is also reported that depletion of microbiota by antibiotics ameliorated IMQ-treated psoriasis in mice, suggesting the role of microbiota in IMQ-induced skin inflammation ${ }^{22,28}$. Furthermore, treatment with IMQ caused altered composition of gut microbiota in mice ${ }^{29}$. Moreover, mice treated with antibiotics showed higher abundance of the genus Lactobacillus in the intestine and on the $\operatorname{skin}^{30}$.

Interestingly, it is recognized that the skin neuroendocrine system acts by preserving and maintaining the skin structural and functional integrity ${ }^{31}$, and that the skin is a sensory organ endowed with neuroendocrine activities $^{32}$. However, as far as we know, there are no reports showing altered composition of microbes in the intestine and on the skin of IMQ-treated mice.

\footnotetext{
${ }^{1}$ Department of Dermatology, Chiba University Graduate School of Medicine, Chiba 260-8670, Japan. ${ }^{2}$ Division of Clinical Neuroscience, Chiba University Center for Forensic Mental Health, Chiba 260-8670, Japan. ${ }^{3}$ Department of Respirology, Chiba University Graduate School of Medicine, Chiba 260-8670, Japan. ${ }^{4}$ Key Laboratory of Medical Electrophysiology of Ministry of Education and Medical Electrophysiological Key Laboratory of Sichuan Province, Collaborative Innovation Center for Prevention and Treatment of Cardiovascular Disease, Institute of Cardiovascular Research, Southwest Medical University, Luzhou 646000, Sichuan, China. ${ }^{\star}$ email: hashimoto@ faculty.chiba-u.jp
} 
a

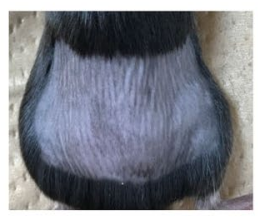

Control

d

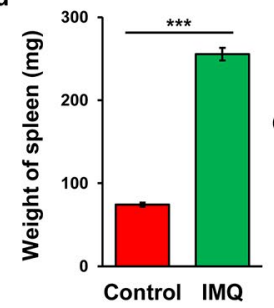

b

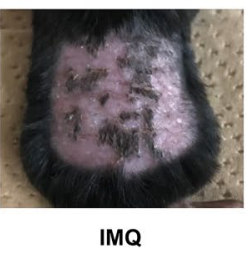

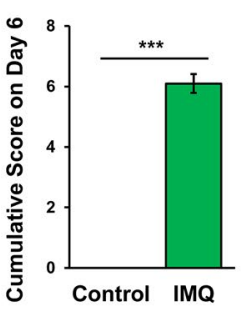

c

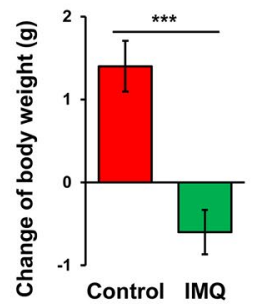

e
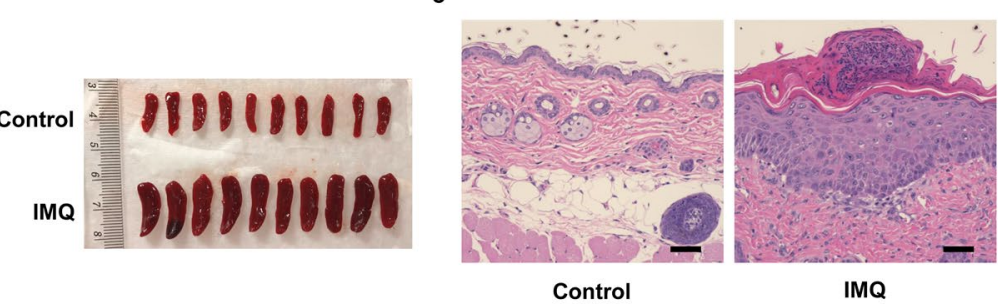

Figure 1. IMQ-induced psoriasis-like model. (a) Mice were treated topically with 5\% IMQ cream or control cream on the shaved backs for five consecutive days. The representative photos of back skin from the two groups. (b) Cumulative score on day 6 of two groups $(P<0.001)$. (c) Change of body weight for the two groups $(P<0.001)$. (d) Weight of spleen $(P<0.001)$. The photos of spleen from the control and IMQ group. (e) HE staining of the back skin of the two groups. Scale bar $=50 \mu \mathrm{m}$. The values represent the mean \pm S.E.M. $(n=10)$. ${ }_{* * *}^{*} P<0.001$.

The present study was undertaken to examine whether topical application of IMQ to skin could influence the composition of microbiota in the intestine and on the skin of adult mice. Using 16S rRNA sequencing, we analyzed the composition of microbiota in the intestine and on the skin of control and IMQ-treated mice. It is well known that short chain fatty acids (SCFAs), the main metabolites produced by microbiota in the gastrointestinal tract, play an important role in the metabolic functions in human and rodents ${ }^{33-36}$. Therefore, we measured levels of SCFAs (i.e., acetic acid, propionic acid, butyric acid, lactic acid, and succinic acid) in the fecal samples.

\section{Results \\ Effects of IMQ on the psoriasis-like score, body weight changes, spleen weight, and pathol- ogy of back skin. IMQ-treated mice developed psoriasis-like dermatitis compared to control mice (Fig. 1a). Cumulative score of IMQ-treated mice was significantly higher than that of control mice (Fig. 1b). Furthermore, the body weight in the IMQ-treated mice was significantly lower than that of control mice (Fig. 1c). Moreover, the weight of spleen in the IMQ-treated mice was significantly higher than that of control mice (Fig. 1d), con- sistent with previous reports ${ }^{27,37-39}$. Representative H\&E staining in the back skin showed parakeratosis with microabscess, acanthosis and an inflammatory infiltrate of the upper dermis in the IMQ-treated mice (Fig. 1e).}

Composition of gut microbiota. Using alpha- and beta-diversity, we examined the composition of the gut microbiota in fecal samples. Mann-Whitney U-test showed a significant difference in the observed OTUs (Fig. 2a). In contrast, there were no changes for ACE and Shannon indices between the two groups (Fig. 2b,c). Beta diversity based on analysis of similarities (ANOSIM) was used to measure the degree of difference in bacterial community, including principal component analysis (PCA) of the OUT composition and principal coordinate analysis (PCoA) of weighted UniFrac distances. PCA demonstrated a significant difference in the microbiome (ANOSIM, $\mathrm{R}=0.6294, P=0.001$ ) (Fig. 2d). In addition, $\mathrm{PCoA}$ of weighted UniFrac distances indicated a clear separation of IMQ group from control group (ANOSIM, $\mathrm{R}=0.3236, P=0.002$ ) (Fig. $2 \mathrm{e}$ ).

The changes of the abundant taxa were analyzed by the LEfSe algorithm. The color differences illustrated differences in the abundant taxa between the two groups. LEfSe analysis showed that IMQ group produced significant different effects on gut microbiota (Fig. 2f). Five mixed-level phylotypes, including the species Lactobacillus intestinalis, Lactobacillus reuteri, Bacteroides uniformis, the genus Bacteroides, and the family Bacteroidaceae, were identified as potential gut microbial markers for the IMQ group (Fig. 2g).

Composition of the gut microbiota at the taxonomic level. At the phylum, there were no changes between the two groups (Figure S1). At the genus level, the abundance of Bacteroides, Parabacteroides, Staphylococcus, Faecalimonas, and Alistipes was significantly different between the two groups (Figure S2 and Table S1). At the species level, the abundance of Lactobacillus murinus, Lactobacillus intestinalis, Lactobacillus reuteri, Lactobacillus taiwanensis, Bacteroides uniformis, Bacteroides acidifaciens, Bacteroides sartorii, Staphylococcus lentus, and Parabacteroides distasonis was significantly different between the two groups (Figure S3 and Table S2). 
a

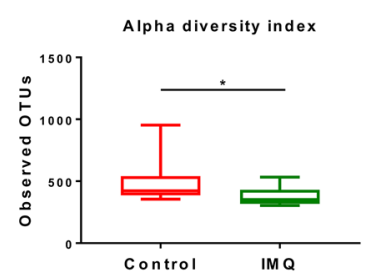

b

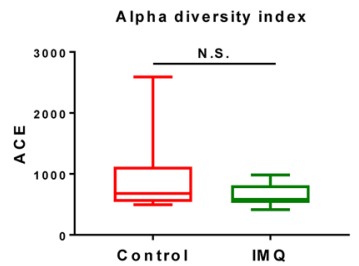

C

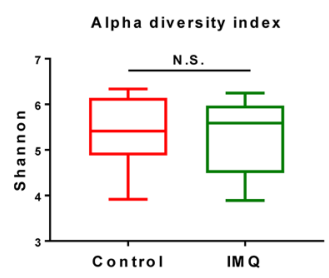

d

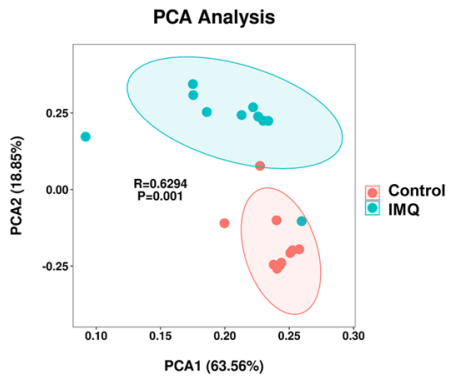

e

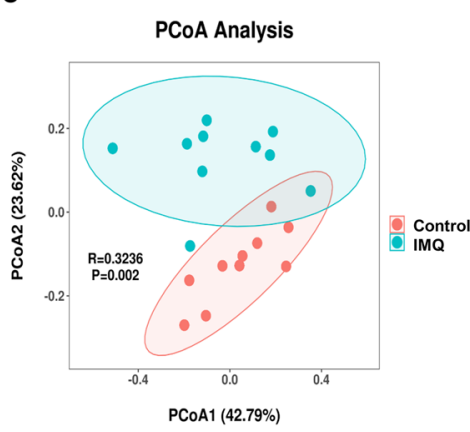

f

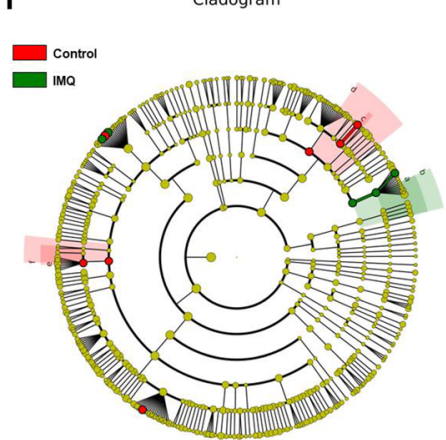

g

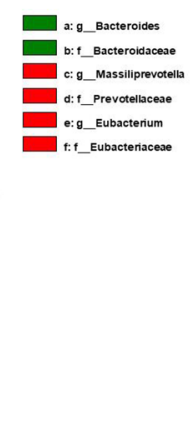

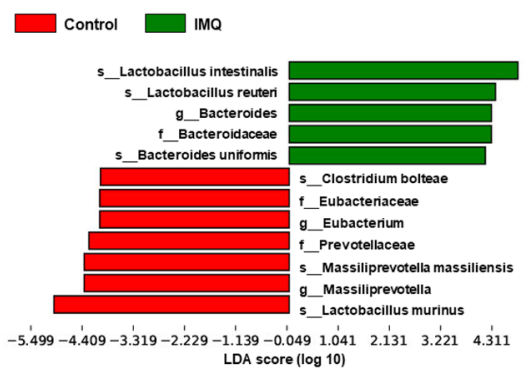

Figure 2. Effects of IMQ on the composition of gut microbiota in alpha-diversity and beta-diversity. (a) Alphadiversity index of observed OTUs (Mann-Whitney $U$ test, $U=21, P=0.029$ ). (b) Alpha-diversity index of ACE (Mann-Whitney U test, $\mathrm{U}=21, P=0.353$ ). (c) Alpha-diversity index of Shannon (Mann-Whitney $\mathrm{U}$ test, $\mathrm{U}=45$, $P=0.739$ ). (d) PCA of beta-diversity based on the OTU level (ANOSIM, $\mathrm{R}=0.6294, P=0.001$ ). Each point represents a single sample which is color-coded according to group, and the two principal components (PC1 and PC2) explained $63.56 \%$ and $18.85 \%$. (e) PCoA plot using weighted UniFrac distance (ANOSIM, R=0.3236, $P=0.002)$. Each point represents a single sample and the two principal components (PCoA1 and PCoA2) explained $42.79 \%$ and $23.62 \%$. (f) LEfSe cladogram (LDA score $>4.0, P<0.05$ ) indicated differentially abundant taxa between control group and IMQ group. Each circle represents the taxonomic categories from the species level as the outermost circle to phylum level as the innermost cycle. (g) Histograms revealed differentially abundant taxa with LDA score $(\log 10)>4.0$ and $P<0.05$ between the control group and IMQ group. The LDA scores of the control group was negative, while those of the IMQ group was positive. The values represent the mean \pm S.E.M. $(\mathrm{n}=10) .{ }^{\star} P<0.05$. N.S.: not significant.

Composition of skin microbiota. The composition of the microbiota on the back skin was analyzed. Mann-Whitney U-test showed significant differences in the observed OTUs, ACE, and Shannon (Fig. 3a-c). Beta-diversity analysis using PCA demonstrated significant differences between IMQ group and control group (ANOSIM, $\mathrm{R}=1.000, P=0.001$ ) (Fig. 3d). Furthermore, significant separation was observed between the two groups on PCoA of weighted UniFrac distances (ANOSIM, $\mathrm{R}=0.7293, P=0.001$ ) (Fig. 3e).

LEfSe difference analysis showed that IMQ group produced significant differential effects on skin microbiota (Fig. 3f). Six mixed-level phylotypes, including the species Staphylococcus lentus, the genus Staphylococcus, the family Staphylcoccaceae, the order Bacillales, the class Bacilli, and the phylum Firmicutes were identified as potential skin microbial markers for the IMQ group (Fig. $3 \mathrm{~g}$ ).

Composition of the skin microbiota at the taxonomic level. At the phylum level, the abundance of Firmicutes, Proteobacteria, Cyanobacteria, Actinobacteria, and Bacteroidetes was significantly different between the two groups (Figure S4 and Table S3). At the genus level, the most abundant bacteria on the skin of IMQtreated mice was Staphylococcus (the mean $=98.5 \%$ ) whereas the level of Staphylococcus on the skin of control mice was low (the mean $=16.2 \%$ ) (Figure S5). Furthermore, the abundance of sixteen bacteria was significantly different between the two groups (Figure S5 and Table S4). At the species level, the most abundant bacteria on the skin of IMQ-treated mice was Staphylococcus lentus (the mean is 93.5\%) whereas the level of Staphylococcus lentus on the skin of control mice was very low (the mean is 7.14\%) (Figure S6). Furthermore, the abundance of fourteen bacteria were significantly different between the two groups (Figure S6 and Table S5).

Correlations between the skin microbiota and the gut microbiota. At the species level, we found several microbes which were significantly altered in the intestine and on the skin between the two groups (Tables S2 and S5). Interestingly, there were negative correlations for Lactobacillus intestinalis, Lactobacillus reuteri, and Lactobacillus taiwanensis between the intestine and the skin (Fig. $4 \mathrm{a}-\mathrm{c}$ ). In contrast, there was a positive correlation for Staphylococcus lentus between the intestine and the skin (Fig. 4d). 
a

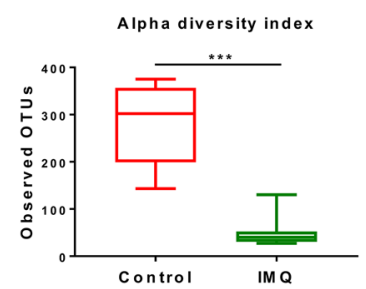

e

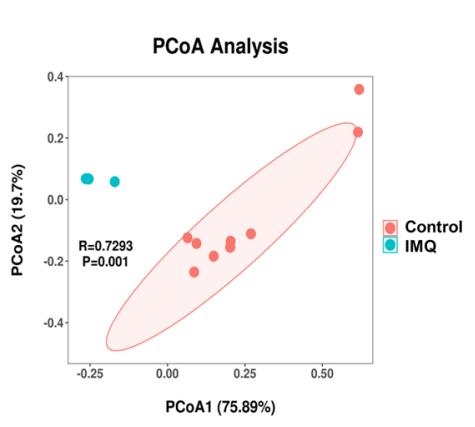

b

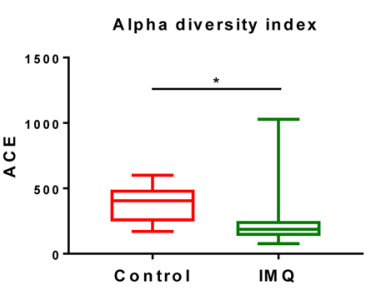

$\mathbf{f}$

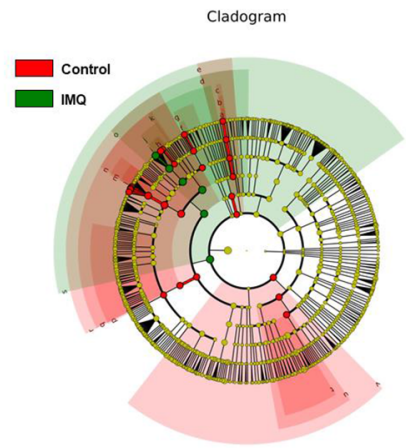

C

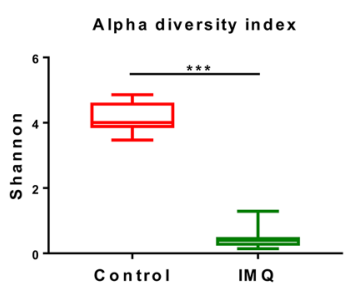

d

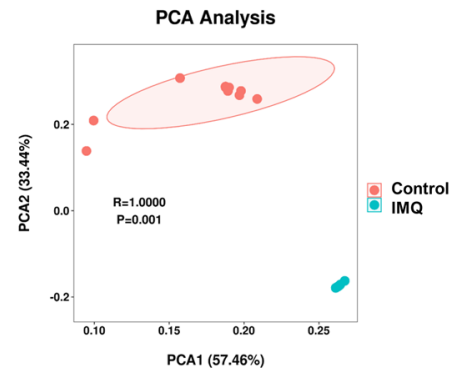

g

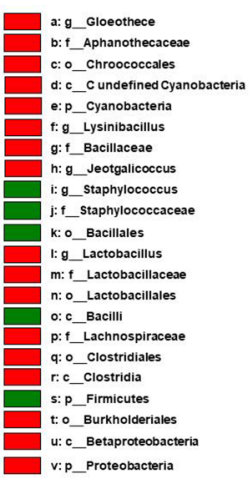

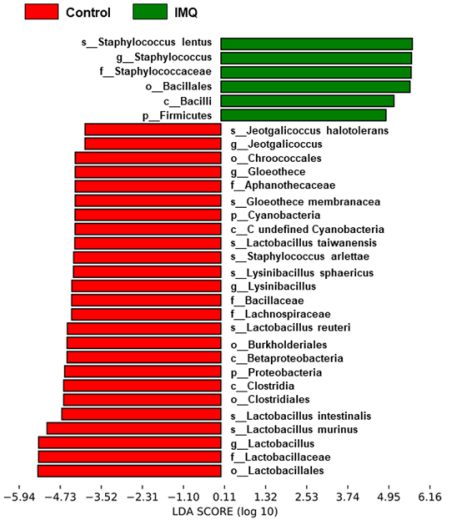

Figure 3. Effects of IMQ on the composition of skin microbiota in alpha-diversity and beta-diversity. (a) Alpha-diversity index of observed OTUs (Mann-Whitney $U$ test, $U=0, P=0.000$ ). (b) Alpha-diversity index of ACE (Mann-Whitney $U$ test, $U=20, P=0.023$ ). (c) Alpha-diversity index of Shannon (Mann-Whitney $U$ test, $\mathrm{U}=0, P=0.000$ ). (d) PCA of beta-diversity based on the OTU level (ANOSIM, $\mathrm{R}=1.000, P=0.001$ ). Each point represents a single sample color-coded according to group, and the two principal components (PC1 and PC2) explained $57.46 \%$ and $33.44 \%$. (e) PCoA plot using upon weighted UniFrac distance (ANOSIM, R=0.7293, $P=0.001$ ). Each point represents a single sample and the two principal components (PCoA1 and PCoA2) explained $75.89 \%$ and $19.7 \%$. (f) LEfSe cladogram (LDA score $>4.0, P<0.05$ ) indicated differentially abundant taxa between control group and IMQ group. Each circle represents the taxonomic categories from the species level as the outermost circle to phylum level as the innermost cycle. (g) Histograms revealed differentially abundant taxa with LDA score $(\log 10)>4.0$ and $P<0.05$ between the control group and IMQ group. The LDA scores of the control group was negative, while those of the IMQ group was positive. The values represent the mean \pm S.E.M. $(\mathrm{n}=10) .{ }^{\star} P<0.05 ;{ }^{* *} P<0.001$.

SCFAs in fecal samples and their correlations with the relative bacterial abundance. The levels of succinic acid and lactic acid in the IMQ group were significantly higher than the control groups (Table 1). In contrast, there were no changes for acetic acid, propionic acid, and n-butyric acid between the two groups (Table 1).

Next, we examined the possible correlations between the relative abundance of microbes and SCFA levels in fecal samples. The succinic acid was significantly correlated with the relative abundance of the genus Parabacteroides ( $\mathrm{r}=-0.464, P=0.0393$ ) (Fig. 5a), the genus Staphylococcus $(\mathrm{r}=0.4981, P=0.0254)$ (Fig. $5 \mathrm{~b})$, the species Parabacteroides distasonis $(\mathrm{r}=-0.485, P=0.0302)$ (Fig. $5 \mathrm{c})$, the species Staphylococcus lentus $(\mathrm{r}=0.4807$, $P=0.0319$ ) (Fig. 5d) and the species Lactobacillus intestinalis $(\mathrm{r}=0.4492, P=0.0469)$ (Fig. 5e) in the two groups. A significant negative correlation between the relative abundance of the genus Parabacteroides $(\mathrm{r}=-0.5079$, $P=0.0222$ ) and lactic acid was observed in two groups (Fig. 5f).

Predictive functional metagenomes. In gut microbiota, two pathways on KEGG level 2, including cardiovascular disease, and endocrine and metabolic disease were significantly different between the two groups (Fig. 6). In skin microbiota, four pathways on KEGG level 3, including sphingolipid signaling pathway, coronavirus disease (COVID-19), steroid degradation, and renin secretion were significantly different between the two groups (Fig. 7).

\section{Discussion}

The major findings of this study were as follows. First, topical application of IMQ to skin caused psoriasis-like phenotypes in mice. Second, IMQ caused significant alterations in the alpha- and beta-diversity of the microbiota in the intestine and on the skin. The LEfSe algorithm of gut microbiota identified the species Lactobacillus intestinalis, Lactobacillus reuteri, and Bacteroides uniformis as potential gut microbial markers for the IMQ group. Furthermore, the LEfSe algorithm of skin microbiota identified the species Staphylococcus lentus as potential skin microbial marker for the IMQ group. Interestingly, correlations for several microbes between the intestine and the skin were observed, suggesting a role of skin-gut-microbiota in IMQ-treated mice. Third, levels of succinic 
a

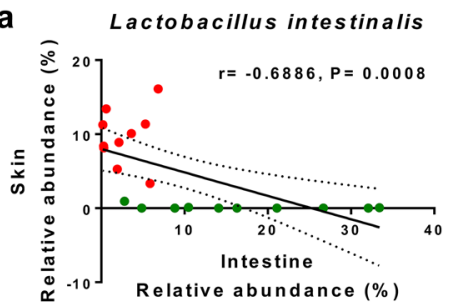

C

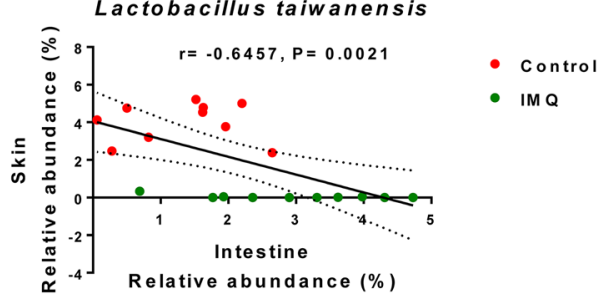

- Control

- IM Q b

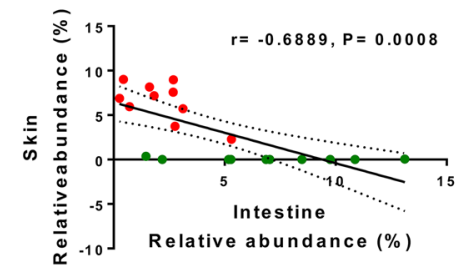

d

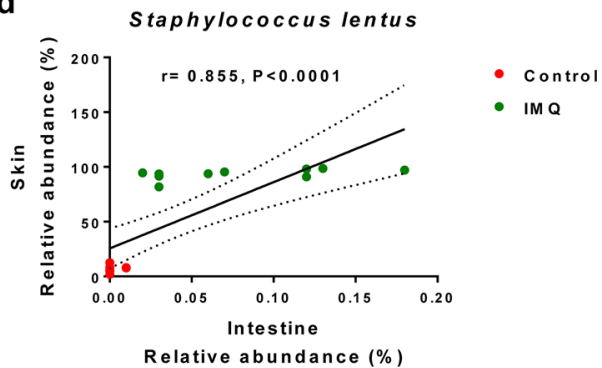

Figure 4. The correlations between bacterial relative abundance in the intestine and skin. (a) There was a significant negative correlation $(\mathrm{r}=-0.6886, P=0.0008)$ of the relative abundance of the species Lactobacillus intestinalis between the intestine and the skin. (b) There was a significant negative correlation $(\mathrm{r}=-0.6889$, $P=0.0008)$ of the relative abundance of the species Lactobacillus reuteri between the intestine and the skin. (c) There was a significant negative correlation $(\mathrm{r}=-0.6457, P=0.0021)$ of the relative abundance of the species Lactobacillus taiwanensis between the intestine and the skin. (d) There was a significant positive correlation $(\mathrm{r}=0.855, P<0.0001)$ of the relative abundance of the species Staphylococcus lentus between the intestine and the skin. The values represent the mean \pm S.E.M. $(n=10)$.

\begin{tabular}{|l|l|l|l|}
\hline SCFAs $(\mathbf{m g} / \mathbf{g})$ & Control & IMQ & Student's $\mathrm{t}$-test \\
\hline Succinic acid & $\mathbf{0 . 1 4 6} \pm \mathbf{0 . 0 2 0}$ & $\mathbf{0 . 3 3 5} \pm \mathbf{0 . 0 6 5}$ & $\mathbf{d f}=\mathbf{1 8}, \mathbf{t}=-\mathbf{2 . 7 6 7}, \boldsymbol{P}=\mathbf{0 . 0 1 3}$ \\
\hline Lactic acid & $\mathbf{1 . 1 4 1} \pm \mathbf{0 . 1 0 7}$ & $\mathbf{1 . 8 3 5} \pm \mathbf{0 . 2 8 9}$ & $\mathbf{d f}=\mathbf{1 8}, \mathbf{t}=-\mathbf{2 . 2 5 2}, \boldsymbol{P}=\mathbf{0 . 0 3 7}$ \\
\hline Acetic acid & $1.877 \pm 0.341$ & $1.750 \pm 0.130$ & $\mathrm{df}=18, \mathrm{t}=0.346, P=0.733$ \\
\hline Propionic acid & $0.299 \pm 0.033$ & $0.372 \pm 0.027$ & $\mathrm{df}=18, \mathrm{t}=-1.704, P=0.106$ \\
\hline n-butyric acid & $0.865 \pm 0.225$ & $0.907 \pm 0.209$ & $\mathrm{df}=18, \mathrm{t}=-0.137, P=0.893$ \\
\hline
\end{tabular}

Table 1. Levels of short-chain fatty acids (SCFAs) in the fecal samples. The values are the mean \pm S.E.M. $(\mathrm{n}=10)$. Bold was statistically significant.

acid and lactic acid in feces increased in the IMQ group compared to control group. Interestingly, we found that the levels of succinic acid (or lactic acid) were correlated with the relative abundance of several microbes in the fecal samples. Finally, the predictive functional analysis of the microbiota of gut and skin showed that IMQ caused alterations in several KEGG pathways. Taken all together, the present data show that topical treatment with IMQ alters the composition of the microbiota in the intestine and on the skin of host.

At the species level, the three Lactobacillus microbes such as Lactobacillus intestinalis, Lactobacillus reuteri, and Lactobacillus taiwanensis were significantly higher in the intestine of IMQ-treated mice compared to control mice. A recent study showed that Lactobacillus intestinalis and Lactobacillus reuteri may be responsible for the depression-like behavior in mice after transplantation of "depression-related microbes" ${ }^{40}$. The increased abundance of Lactobacillus intestinalis, Lactobacillus reuteri and Lactobacillus taiwanensis by IMQ treatment may contribute to the increased levels of lactic acid in the IMQ-treated mice since Lactobacilli ferment lactose into lactic acid ${ }^{41}$. Collectively, it is likely that increased abundance of Lactobacillus bacteria may contribute to increased levels of lactic acid in the host gut although further detailed study is needed. Considering the beneficial actions of Lactobacillus reuteri on the host immune system ${ }^{41,42}$, it seems that IMQ-induced increases in the abundance of bacteria might reflect a compensatory response in the host. There are many species of Lactobacillus which may have beneficial and harmful effects in the host ${ }^{43}$. Nonetheless, future studies are needed to investigate the mechanisms underpinning increases of these Lactobacillus bacteria in the intestine of IMQ-treated mice.

Furthermore, we found significant differences for Bacteroides uniformis, Bacteroides acidifaciens, Bacteroides sartorii, Staphylococcus lentus and Parabacteroides distasonis in the intestine between the two groups. As far as we know, there are no reports showing alterations in these three Bacteroides bacteria in IMQ-treated mice and patients with psoriasis. Pretreatment with the antibiotic metronidazole increased the abundance of 
a
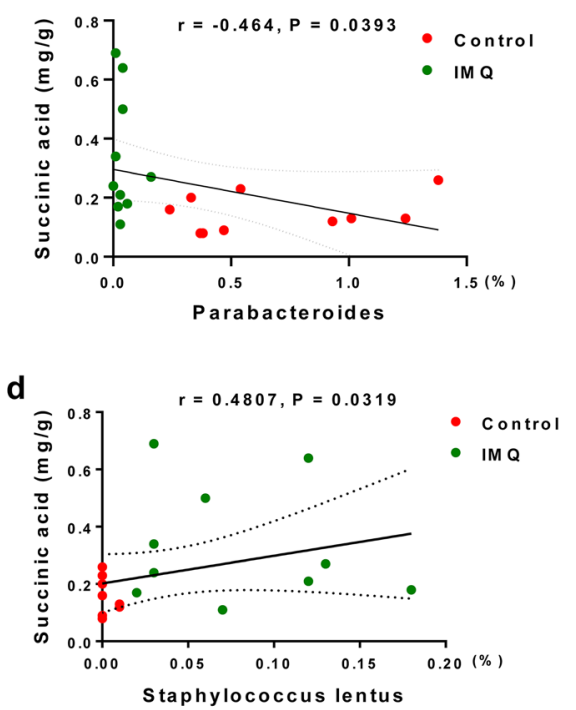

b

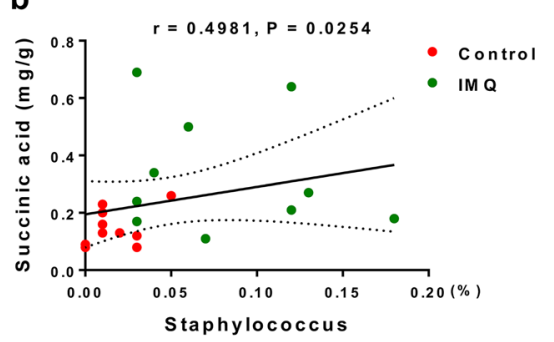

e

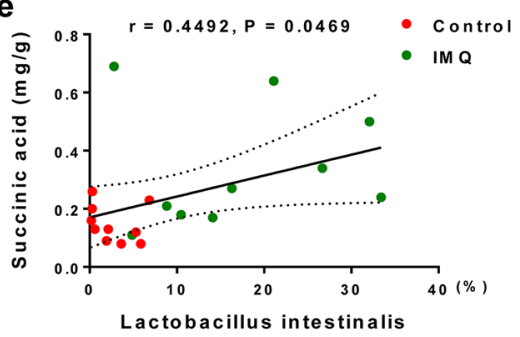

C

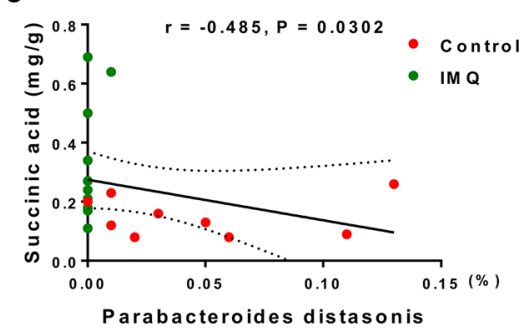

f

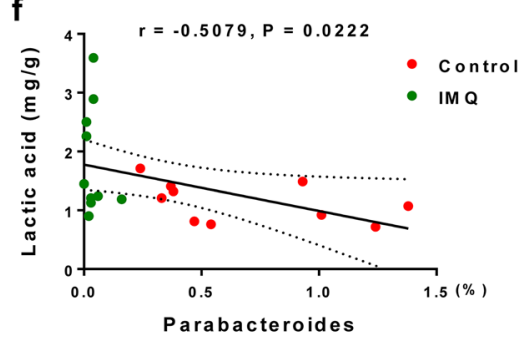

Figure 5. The correlations between bacterial relative abundance and SCFAs. (a) A significant negative correlation ( $\mathrm{r}=-0.464, P=0.0393$ ) between the relative abundance of the genus Parabacteroides and succinic acid was shown in the control group and IMQ group. (b) A significant positive correlation $(r=0.4981$, $P=0.0254$ ) between the relative abundance of the genus Staphylococcus and succinic acid was shown in the two groups. (c) A significant negative correlation $(r=-0.485, P=0.0302)$ between the relative abundance of the species Parabacteroides distasonis and succinic acid was shown in the two groups. (d) A significant positive correlation $(\mathrm{r}=0.4807, P=0.0319)$ between the relative abundance of the species Staphylococcus lentus and succinic acid in the two groups. (e) A significant positive correlation $(r=0.4492, P=0.0469)$ between the relative abundance of the species Lactobacillus intestinalis and succinic acid in the two groups. (f) There was a significant negative correlation $(\mathrm{r}=-0.5079, P=0.0222)$ between the relative abundance of the genus Parabacteroides and lactic acid in the two groups. The values represent the mean \pm S.E.M. $(n=10)$.

Parabacteroides distasonis in the intestine of IMQ-treated mice ${ }^{30}$. In contrast, Parabacteroides distasonis were significantly decreased in the patients with psoriasis ${ }^{44}$.

At the species level, we found many skin microbes which altered in the IMQ-treated mice (Table S5). Importantly, the most abundant microbe on the skin from IMQ-treated mice was Staphylococcus lentus, and the abundance of Staphylococcus lentus in the IMQ-treated mice were significantly higher than control mice (Table S5). Staphylococcus lentus are commensal bacterium colonizing the skin of animals and has been associated with infections in animals ${ }^{45}$. It seems that high abundance of Staphylococcus lentus may play a role in IMQ-induced psoriasis-like symptoms in mice. However, the precise mechanisms underlying high abundance of Staphylococcus lentus on the skin of IMQ-treated mice are currently unclear. Further study is needed to examine the role of Staphylococcus lentus in psoriasis-like symptom of IMQ-treated mice.

In this study, we found several microbes which altered in the both intestine and skin. Interestingly, we found significant correlations for Lactobacillus intestinalis, Lactobacillus reuteri, Lactobacillus taiwanensis, and Staphylococcus lentus between the intestine and the skin. To the best of our knowledge, this is the first report showing the correlations for microbes in both the intestine and the skin, supporting the skin-gut microbiota axis ${ }^{46,47}$. From the current data, it is unclear whether changes in gut microbiota can affect skin microbiota or these two phenomena are independent. In this study, we found that topical treatment with IMQ caused increased volume of spleen through systemic inflammation, resulting in abnormal changes in the microbiota composition in the intestine and on the skin of mice. Although the precise mechanisms underlying the association between the skin and the intestine remain unclear, the current data strongly suggest a role of skin-gut microbiota axis in IMQtreated mice. Therefore, it is of great interest to investigate whether the composition of microbiota in the intestine and on the skin from patients with psoriasis is altered compared to healthy control subjects.

Succinic acid is produced in large amounts during bacterial fermentation of dietary fiber, and it is considered as a key intermediate in the synthesis of propionic acid ${ }^{48,49}$. Interestingly, germ-free mice have little or no detectable levels of succinic acid in feces compared to conventional mice, indicating that gut microbiota are the predominant source for succinic acid. Higher levels of succinic acid may be related with high abundance of Bacteroides in fecal samples since succinic acid is produced by primary fermenters such as Bacteroides ${ }^{49}$. It is also shown that elevated levels of succinic acid in the feces are associated with intestinal inflammation ${ }^{48,49}$. Collectively, higher levels of succinic acid in the feces of IMQ-treated mice might be associated with intestinal inflammation although further study is needed.

To understand the role of altered composition of microbiota in the intestine and skin of IMQ-treated mice, we examined the predictable function of the microbiota. The current data show that IMQ treatment may contribute to the altered metabolism (i.e., cardiovascular disease, and endocrine and metabolic disease) induced by gut microbiota. In addition, the current data show that IMQ treatment could produce the altered metabolism (i.e., sphingolipid signaling pathway, coronavirus disease-COVID-19, steroid degradation, and renin secretion) 
A

A
Cellular Processes
Environmental
Information Processing
Genetic Information
Processing

Human Diseases

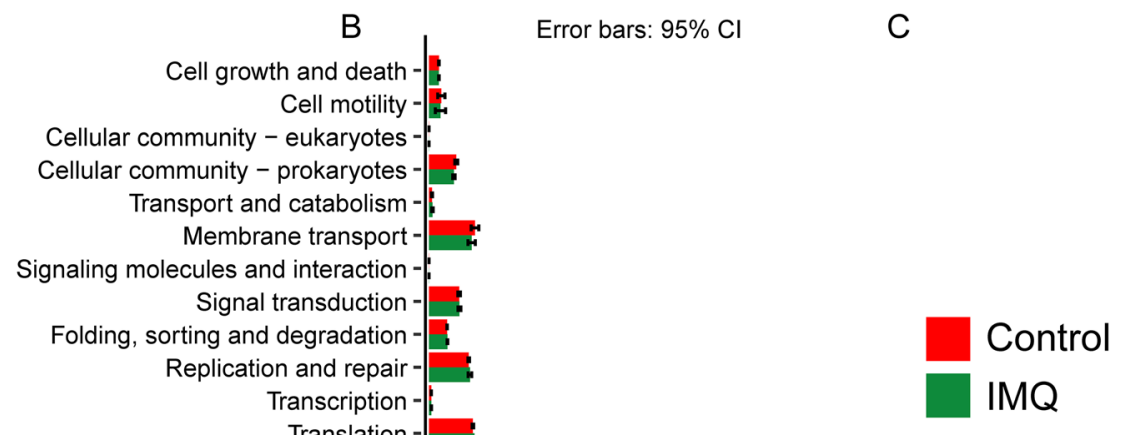

Organismal Systems

Translation -

Cancer: overview -,

Cancer: specific types -

Cardiovascular disease - *

Drug resistance: antimicrobial -

Drug resistance: antineoplastic -

Endocrine and metabolic disease - *

Immune disease -

Infectious disease: bacterial -

Infectious disease: parasitic -

Infectious disease: viral -

Neurodegenerative disease -

Substance dependence -

Amino acid metabolism -

Biosynthesis of other secondary metabolites -

Carbohydrate metabolism -

Energy metabolism -

Global and overview maps -

Glycan biosynthesis and metabolism Lipid metabolism -

Metabolism of cofactors and vitamins -

Metabolism of other amino acids Metabolism of terpenoids and polyketides -

Nucleotide metabolism -

Xenobiotics biodegradation and metabolism -

Aging -

Circulatory system -

Development and regeneration -

Digestive system -

Endocrine system -

Environmental adaptation - ";

Excretory system -

Immune system -

Nervous system -

\section{$\begin{array}{llll}\text { KEGG_Pathways Levels } 1 \quad \text { KEGG_Pathways Levels } 2 & 0.0 & 0.2 & 0.4 \\ & & \text { Relative abundance of metagenomes }\end{array}$}

Figure 6. Relative abundance of KEGG pathways of functional categories in the gut microbiota. Functional predictions of the gut microbiota between the control group and IMQ group. Significant differences of KEGG pathways at level 2 were detected using STAMP software based on the KEGG pathway database (www.kegg.jp/ kegg1.html). ${ }^{\star} P<0.05$.

induced by skin microbiota. It is noteworthy that we could detect the altered metabolism of endocrine disease in IMQ-treated mice since the neuroendocrine system plays a role in the skin function ${ }^{31,32}$. Furthermore, it is likely that gut microbiota is more complex than skin microbiota since we detected many pathways for gut microbiota compared to skin microbiota. Taken together, it is likely that these KEGG pathways provide a new functional view for understanding the gut and skin microbiota that contribute to psoriasis-like symptoms. Finally, this study has a potential limitation. A future study using antibiotic cocktail is needed to ascertain the correlation between skin microbiota and gut microbiota in IMQ-treated mice.

In conclusion, this study shows that topical treatment with IMQ causes abnormal changes in the microbiota composition in the intestine and on the skin of adult mice, and that levels of succinic acid were associated with the relative abundance of several microbes. Furthermore, we found correlations for several microbes between the intestine and the skin, suggesting a role of skin-gut microbiota in psoriasis. 


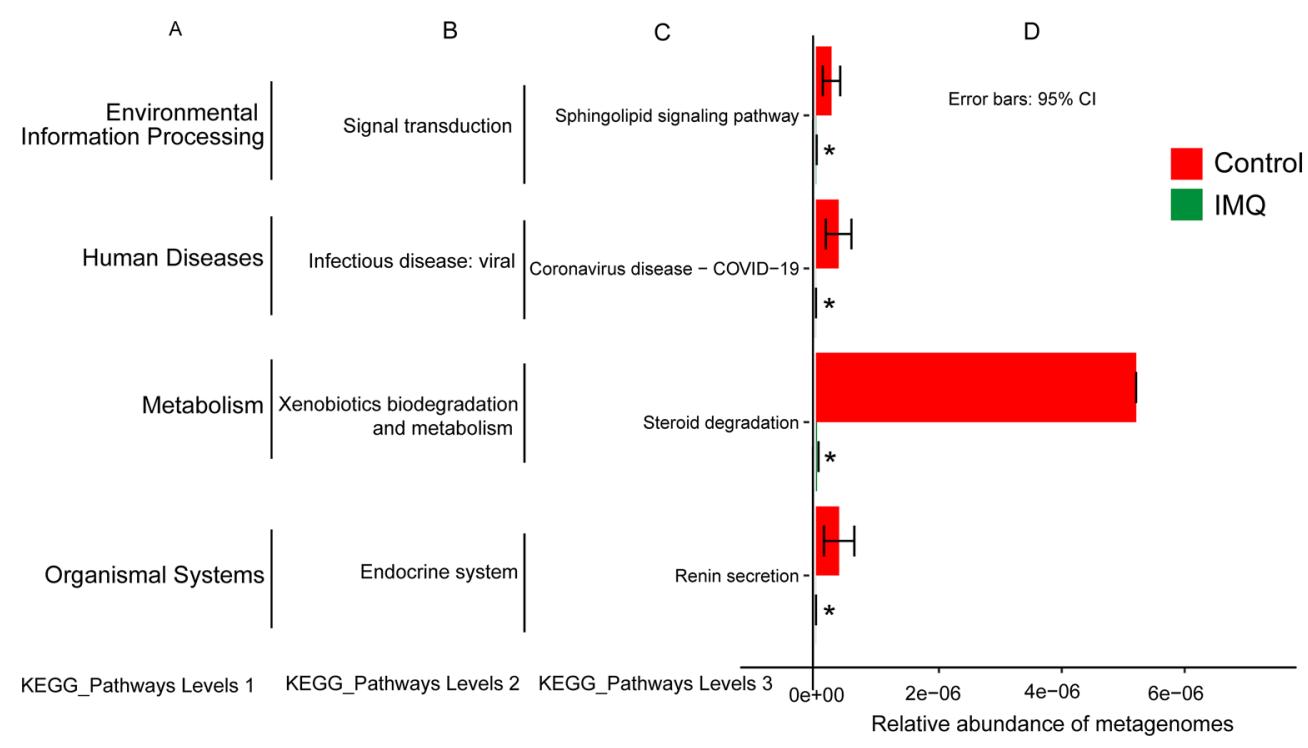

Figure 7. Relative abundance of KEGG pathways of functional categories in the skin microbiota. Functional predictions of the skin microbiota between the control group and IMQ group. Significant differences of KEGG pathways at level 3 were detected using STAMP software based on the KEGG pathway database (www.kegg.jp/ keggl.html). ${ }^{\star} P<0.05$.

\section{Materials and methods}

Animals. Female C57BL/6 mice (9 weeks old, weighing 18-21 g, $\mathrm{n}=20$, Japan SLC Inc., Hamamatsu, Shizuoka, Japan) were used. Mice were housed (5 per cage) under a 12-h/12-h light/dark cycle (lights on between 07:00 and 19:00), with ad libitum access to food access to food (CE-2; CLEA Japan, Inc., Tokyo, Japan) and water. The experimental protocol was approved by Chiba University Institutional Animal Care and Use Committee (Permission number: 2-433) ${ }^{50}$. This study was carried out in strict accordance with the recommendations in the Guide for the Care and Use of Laboratory Animals of the National Institutes of Health, USA ${ }^{50}$. This study was also carried out in compliance with the ARRIVE guidelines. All efforts were made to minimize animal suffering.

Treatment of IMQ and collection of samples. The shaved back skin of mice was treated with $62.5 \mathrm{mg}$ of 5\% IMQ cream (Beselna cream; Mochida Pharmaceutical Co., Tokyo, Japan) daily for 5 consecutive days. Control mice were treated similarly with $62.5 \mathrm{mg}$ of white petrolatum (Maruishi Pharmaceutical Co., Osaka, Japan). Skin, fecal and spleen samples were collected on day 6. The clinical skin score was measured on day 1 and day 6. The degree of skin inflammation was scored by cumulative disease severity score, similar to the human Psoriasis Area and Severity Index, but not taking the area into account. Erythema, scaling, and thickening were scored independently from 0 to 4 : 0 , none; 1 , slight; 2 , moderate; 3 , marked; 4 , very marked. The single scores were summed, resulting in a theoretical maximal total score of $12^{27}$.

Fresh fecal samples of mice were collected from 7:30 to 8:30 on day 6 to exclude any circadian effects on the microbes. The fecal samples were placed into sterilized screw-cap microtubes immediately after defecation, and they were frozen in liquid-nitrogen immediately. The samples were stored at $-80{ }^{\circ} \mathrm{C}$ until use $\mathrm{e}^{51}$.

Skin swabs from the shaved back skin of IMQ-treated mice or control mice were put into extraction tube containing a solution $(0.15 \mathrm{M} \mathrm{NaCl}$ and $0.1 \%$ Tween 20$)$ and rotated for at least 20 times. After squeezing as much liquid as possible from the swab by pushing the swabs against the sides of the tubes, the tubes were stored at $-80^{\circ} \mathrm{C}$ until analysis.

Histology. Back skin samples from control and IMQ-treated groups were collected and fixed in $10 \%$ formalin (FUJIFILM Wako Pure Chemical Corp.). Staining with hematoxylin and eosin (HE) was performed at Biopathology Institute Co., Ltd (Kunisaki, Oita, Japan). Back skin samples were embedded in paraffin, and sections of $3 \mu \mathrm{m}$ were prepared and subjected to HE staining. Representative images of two groups were obtained using a Keyence BZ-9000 Generation II microscope (Osaka, Japan).

16S rRNA analysis. The DNA extractions from the fecal and skin samples and 16S rRNA sequencing analyses were performed by MyMetagenome Co., Ltd. (Tokyo, Japan), as reported previously ${ }^{40,50-52}$. DNA extraction from mouse samples and purification were performed according to the method of the previous report ${ }^{53,54}$. The $16 \mathrm{~S}$ rRNA analysis of samples was performed as previously reported ${ }^{53,54}$. Briefly, PCR was performed using 27Fmod 5'-AGRGTTTGATYMTGGCTCAG-3' and 338R 5'-TGCTGCCTCCCGTAGGAGT-3' to amplify the V1-V2 region of the bacterial 16S rRNA gene. The $16 \mathrm{~S}$ amplicons were then sequenced using MiSeq according to the Illumina protocol. Taxonomic assignment of OTUs was made by similarity searches against the Ribo- 
somal Database Project and the National Center for Biotechnology Information genome database using the GLSEARCH program.

Alpha diversity was used to analyze the species diversity, composed of richness and evenness, within a sample through three indices including the observed OTU, ACE, and Shannon indices ${ }^{55}$. Beta diversity was used to measure differences of species diversity among samples, including PCA and PCoA with ANOSIM. Differences in bacterial taxa between groups at the species or higher level (depending on the taxon annotation) were calculated based on linear discriminant analysis (LDA) effect size (LEfSe) using LEfSe software (LDA score $>4.0, P<0.05$ ) (https://www.omicstudio.cn/tool/) $)^{56}$.

Prediction of functional profiles of gut microbiota using PICRUSt. Using the 16S rRNA gene sequencing data and KEGG (Kyoto Encyclopedia of Genes and Genome) orthology (http://www.kegg.jp/kegg1. html) ${ }^{57}$, we performed PICRUSt (Phylogenetic Investigation of Communities by Reconstruction of Unobserved States) analysis and STAMP (Statistical Analysis of Metagenomic Profiles) software v2.1.3 (http://kiwi.cs.dal.ca/ Software/STAMP) for the functional prediction of microbiota in the intestine and $\operatorname{skin}^{55,58,59}$.

Measurement of short-chain fatty acid (SCFA) levels. Concentrations of SCFAs (i.e., acetic acid, propionic acid, butyric acid, lactic acid, succinic acid) in fecal samples were measured at TechnoSuruga Laboratory, Co., Ltd. (Shizuoka, Japan), as reported previously ${ }^{50,51,55,60}$. The data of SCFAs were shown as milligrams per gram of feces.

Statistical analysis. Data are shown as the mean \pm standard error of the mean (S.E.M.). Alpha-diversity of the gut and skin microbiota were analyzed using Mann-Whitney U-test. Analysis of beta-diversity of the gut and skin microbiota including PCA of OTU level and PCoA of weighted UniFrac distances were performed based on ANOSIM by R package vegan (2.5.4) (https://CRAN.R-project.org/package=vegan ${ }^{61}$. Data for SCFA levels were analyzed using Student t-test. Correlations between SCFAs and the relative bacterial abundance were analyzed using Spearman's correlation analysis. Correlations between the relative abundance of bacteria in the skin and intestine were also analyzed using Spearman's correlation analysis. $P<0.05$ was considered statistically significant.

\section{Data availability}

The data that support the findings of this study are available from the corresponding author upon reasonable request.

Received: 30 January 2021; Accepted: 12 May 2021

Published online: 28 May 2021

\section{References}

1. Michalek, I. M., Loring, B. \& John, S. M. A systematic review of worldwide epidemiology of psoriasis. J. Eur. Acad. Dermatol. Venereol. 31, 205-212 (2017).

2. Boehncke, W. H. \& Schön, M. P. Psoriasis. Lancet 386, 983-994 (2015).

3. Armstrong, A. W. \& Read, C. Pathophysiology, clinical presentation, and treatment of psoriasis: a review. JAMA 323, 1945-1960 (2020).

4. Geale, K., Henriksson, M., Jokinen, J. \& Schmitt-Egenolf, M. Association of skin psoriasis and somatic comorbidity with the development of psychiatric illness in a Nationwide Swedish Study. JAMA Dermatol. 156, 795-804 (2020).

5. Kleyn, C. E. et al. Psoriasis and mental health workshop report: exploring the links between psychosocial factors, psoriasis, neuroinflammation and cardiovascular disease risk. Acta Derm. Venereol. 100, adv00020 (2020).

6. Nestle, F. O., Kaplan, D. H. \& Barker, J. Psoriasis. N. Engl. J. Med. 361, 496-509 (2009).

7. Round, J. L. \& Mazmanian, S. K. The gut microbiota shapes intestinal immune responses during health and disease. Nat. Rev. Immunol. 9, 313-323 (2009).

8. Cho, I. \& Blaser, M. J. The human microbiome: at the interface of health and disease. Nat. Rev. Genet. 13, 260-272 (2012).

9. Shreiner, A. B., Kao, J. Y. \& Young, V. B. The gut microbiome in health and in disease. Curr. Opin. Gastroenterol. 31, 69-75 (2015).

10. Althani, A. A. et al. Human microbiome and its association with health and diseases. J. Cell Physiol. 231, 1688-1694 (2016).

11. Fung, T. C., Olson, C. A. \& Hsiao, E. Y. Interactions between the microbiota, immune and nervous systems in health and disease. Nat. Neurosci. 20, 145-155 (2017).

12. Young, V. B. The role of microbiome in human health and disease: an introduction for clinicians. BMJ 356, j831 (2017)

13. Cryan, J. F. et al. The microbiota-gut-brain axis. Physiol. Rev. 99, 1877-2013 (2019).

14. Chen, L. et al. Skin and gut microbiome in psoriasis: gaining insight into the pathophysiology of it and finding novel therapeutic strategies. Front. Microbiol. 11, 589726 (2020).

15. Codoñer, F. M. et al. Gut microbial composition in patients with psoriasis. Sci. Rep. 8, 3812 (2018).

16. Hidalgo-Cantabrana, C. et al. Gut microbiota dysbiosis in a cohort of patients with psoriasis. Br. J. Dermatol. 181, 1287-1295 (2019).

17. Shapiro, J. et al. Psoriatic patients have a distinct structural and functional fecal microbiota compared with controls. J. Dermatol. 46, 595-603 (2019).

18. Dei-Cas, I., Giliberto, F., Luce, L., Dopazo, H. \& Penas-Steinhardt, A. Metagenomic analysis of gut microbiota in non-treated plaque psoriasis patients stratified by disease severity: development of a new psoriasis-microbiome index. Sci. Rep. 10, 12754 (2020).

19. Sikora, M. et al. Gut microbiome in psoriasis: An updated review. Pathogens 9, 463 (2020).

20. Fahlén, A., Engstrand, L., Baker, B. S., Powles, A. \& Fry, L. Comparison of bactrial microbiota in skin biosies from normal and psoriatic skin. Arch. Dermatol. Res. 304, 15-22 (2012).

21. Alekseyenko, A. V. et al. Community differentiation of the cutaneous microbiota in psoriasis. Microbiome 1, 31 (2013).

22. Zákostelská, Z. et al. Intestinal microbiota promotes psoriasis-like skin inflammation by enhancing Th17 response. PLoS ONE 11, e0159539 (2016).

23. Assarsson, M., Duvetorp, A., Dienus, O., Söderman, J. \& Seifert, O. Significant changes in the skin microbiome in patients with chronic plaque psoriasis after treatment with narrowband ultraviolet B. Acta Derm. Venereol. 98, 428-436 (2018). 
24. Chang, H. W. et al. Alteration of the cutaneous microbiome in psoriasis and potential role in Th17 polarization. Microbiome 6, 154 (2018).

25. Fyhrquist, N. et al. Microbe-host interplay in atopic dermatitis and psoriasis. Nat. Commun. 10, 4703 (2019).

26. Flutter, B. \& Nestle, F. O. TLRs to cytokines: Mechanistic insights from the imiquimod mouse model of psoriasis. Eur. J. Immunol. 43, 3138-3146 (2013).

27. van der Fits, L. et al. Imiquimod-induced psoriasis-like skin inflammation in mice is mediated via the IL-23/IL-17 axis. J. Immunol. 182, 5836-5845 (2009).

28. Zanvit, P. et al. Antibiotics in neonatal life increase murine susceptibility to experimental psoriasis. Nat. Commun. 6, 8424 (2015).

29. Kiyohara, H. et al. Toll-like receptor 7 agonist-induced dermatitis causes severe dextran sulfate sodium colitis by altering the gut microbiome and immune cells. Cell Mol. Gastroenterol. Hepatol. 7, 135-156 (2018).

30. Stehlikova, Z. et al. Crucial role of microbiota in experimental psoriasis revealed by a gnotobiotic mouse model. Front. Microbiol. 10, 236 (2019).

31. Slominski, A. \& Wortsman, J. Neuroendocrinology of the skin. Endocr. Rev. 21, 457-487 (2000).

32. Slominski, A. T., Manna, P. R. \& Tuckey, R. C. On the role of skin in the regulation of local and systemic steroidogenic activities. Steroids 103, 72-88 (2015).

33. den Besten, G. et al. The role of short-chain fatty acids in the interplay between diet, gut microbiota, and host energy metabolism. J. Lipid Res. 54, 2325-2340 (2013).

34. Morrison, D. J. \& Preston, T. Formation of short chain fatty acids by the gut microbiota and their impact on human metabolism. Gut Microbes 7, 189-200 (2016).

35. Chambers, E. S., Preston, T., Frost, G. \& Morrison, D. J. Role of gut microbiota-generated short-chain fatty acids in metabolic and cardiovascular health. Curr. Nutr. Rep. 7, 198-206 (2018).

36. Dalile, B., Van Oudenhove, L., Vervliet, B. \& Verbeke, K. The role of short-chain fatty acids in microbiota-gut-brain communication. Nat. Rev. Gastroenterol. Hepatol. 16, 461-478 (2019).

37. Qin, S. et al. Endogenous n-3 polyunsaturated fatty acids protect against imiquimod-induced psoriasis-like inflammation via the IL-17/IL-23 axis. Mol. Med. Rep. 9, 2097-2104 (2014).

38. Lee, J. et al. Tussilagonone ameliorates psoriatic fearures in keratinocytes and imiquimod-induced psoriasis-like lesions in mice via NRF2 activation. J. Invbestig. Dermatol. 140, 1223-1232 (2020).

39. Schwarz, A., Philippsen, R. \& Schwarz, T. Induction of regulatory T cells and correction of cytokine disbalance by short-chain fatty acids: implications for psoriasis therapy. J. Investig. Dermatol. 141, 95-104 (2021).

40. Wang, S. et al. Ingestion of Lactobacillus intestinalis and Lactobacillus reuteri causes depression- and anhedonia-like phenotypes in antibiotic-treated mice via the vagus nerve. J. Neuroinflamm. 17, 241 (2020).

41. Fine, R. L., Mubiru, D. L. \& Kriegel, M. A. Friend or foe? Lactobacillus in the context of autoimmune disease. Adv. Immunol. 146, $29-56(2020)$.

42. Mu, Q., Tavella, V. J. \& Luo, X. M. Role of Lactobacillus reuteri in human health and diseases. Front. Microbiol. 9,757 (2018).

43. Masood, M. I., Qadir, M. I., Shirazi, J. H. \& Khan, I. U. Beneficial effects of lactic acid bacteria on human being. Crit. Rev. Microbiol. 37, 91-98 (2010)

44. Shapiro, J. et al. Psoriatic patients have a distinct structural and functional fecal microbiota compared with controls. J. Dermatol. 46, 595-603 (2019).

45. Nemeghaire, S. et al. The ecological importance of the Staphylococcus sciuri species group as a reservor for resistance and virulence genes. Vet. Microbiol. 171, 342-356 (2014).

46. Chen, G. et al. Gut-brain-skin axis in psoriasis: a review. Dermatol. Ther (Heidelb) 11, 25-38 (2021).

47. Van Splunter, M. et al. Mechanisms underlying the skin-gut cross talk in the development of IgE-mediated food allergy. Nutrients $12,3830(2020)$.

48. Connors, J., Dawe, N. \& Van Limbergen, J. The role of succinate in the regulation of intestinal inflammation. Nutrients 11, 25 (2018).

49. Fernández-Veledo, S. \& Vendrell, J. Gut microbiota-derived succinate: Friend or foe in human metabolic diseases?. Rev. Endocr. Metab. Disord. 20, 439-447 (2019).

50. Wang, S. et al. Antibiotic-induced microbiome depletion is associated with resilience in mice after chronic social defeat stress. J. Affect. Disord. 260, 448-457 (2020).

51. $\mathrm{Pu}$, Y. et al. A role of the subdiaphragmatic vagus nerve in depression-like phenotypes in mice after fecal microbiota transplantation from Chrna7 knock-out mice with depression-like phenotypes. Brain Behav. Immun. 94, 318-326 (2021).

52. Zhang, J. et al. A key role of the subdiaphragmatic vagus nerve in the depression-like phenotype and abnormal composition of gut microbiota in mice after lipopolysaccharide administration. Transl. Psychiatry 10, 186 (2020).

53. Kim, S. W. et al. Robustness of gut microbiota of healthy adults in response to probiotic intervention revealed by high-throughput pyrosequencing. DNA Res. 20, 241-253 (2013).

54. Shibagaki, N. et al. Aging-related changes in the diversity of women's skin microbiomes associated with oral bacteria. Sci. Rep. 7, 10567 (2017).

55. Wei, Y. et al. Abnormalities of the composition of the gut microbiota and short-chain fatty acids in mice after splenectomy. Brain Beahv. Imun. Health 11, 100198 (2021).

56. Segata, N. et al. Metagenomic biomarker discovery and explanation. Genome Biol. 12, R60 (2011).

57. Kanehisa, M., Furumichi, M., Sato, Y., Ishiguro-Watanabe, M. \& Tanabe, M. KEGG: integrating viruses and cellular organisms. Nucleic Acid Res. 49(D1), D545-D551 (2021).

58. Langille, M. G. et al. Predictive functional profiling of microbial communities using $16 \mathrm{~S}$ rRNA marker gene sequences. Nat. Biotechnol. 31, 814-821 (2013).

59. Parks, D. H., Tyson, G. W., Hugenholtz, P. \& Beiko, R. G. STAMP: statistical analysis of taxonomic and functional profiles. Bioinformatics 30, 3123-3124 (2014).

60. Zhang, K. et al. Abnormal composition of gut microbiota is associated with resilience versus susceptibility to inescapable electric stress. Transl. Psychiatry 9, 231 (2019).

61. Xin, Y. \& Sun, J. Hypothesis testing and statistical analysis of microbiome. Genes Dis. 4, 138-148 (2017).

\section{Acknowledgements}

This study was in part supported by grant-in-Aid for the National Natural Science Foundation of China (NSFC) (to Y.W., 31701009). Dr. Yan Wei was supported by the China Scholarship Council (China). Dr. Lijia Chang was supported by the Japan China Sasakawa Medical Fellowship (Tokyo, Japan).

\section{Author contributions}

H.H. conceived the project, designed the experiments, analyzed the data, and drafted the manuscript. Y.H., Y.F., and T.I. performed the experiments. Y.W. and L.C. performed the analyzed the data of microbiota. H.M. worked 
on the general coordination of the study. K.H. conceived the project, designed the experiments, and wrote the manuscript.

\section{Competing interests}

The authors declare no competing interests.

\section{Additional information}

Supplementary Information The online version contains supplementary material available at https://doi.org/ 10.1038/s41598-021-90480-4.

Correspondence and requests for materials should be addressed to K.H.

Reprints and permissions information is available at www.nature.com/reprints.

Publisher's note Springer Nature remains neutral with regard to jurisdictional claims in published maps and institutional affiliations.

(c) (i) Open Access This article is licensed under a Creative Commons Attribution 4.0 International License, which permits use, sharing, adaptation, distribution and reproduction in any medium or format, as long as you give appropriate credit to the original author(s) and the source, provide a link to the Creative Commons licence, and indicate if changes were made. The images or other third party material in this article are included in the article's Creative Commons licence, unless indicated otherwise in a credit line to the material. If material is not included in the article's Creative Commons licence and your intended use is not permitted by statutory regulation or exceeds the permitted use, you will need to obtain permission directly from the copyright holder. To view a copy of this licence, visit http://creativecommons.org/licenses/by/4.0/.

(C) The Author(s) 2021 